Nervenarzt 2021 · 92:197-198

https://doi.org/10.1007/s00115-020-01043-3

Angenommen: 7. Dezember 2020

(c) Der/die Autor(en) 2021

\author{
Andreas Heinz ${ }^{1}$ Kristina Adorjan ${ }^{2,3,4} \cdot$ Tobias Banaschewski $^{5}$. \\ Gunter Schumann $^{6,7} \cdot$ Michael Rapp 8 \\ 'Klinik für Psychiatrie und Psychotherapie, CCM, Charité - Universitätsmedizin Berlin, Berlin, Deutschland \\ ${ }^{2}$ Klinik für Psychiatrie und Psychotherapie, LMU Klinikum, München, Deutschland \\ ${ }^{3}$ Institut für Psychiatrische Phänomik und Genomik (IPPG), LMU Klinikum, München, Deutschland \\ ${ }^{4}$ Center for International Health $(\mathrm{CIH})$, LMU München, München, Deutschland \\ ${ }^{5}$ Klinik für Psychiatrie und Psychotherapie des Kindes- und Jugendalters, Zentralinstitut für seelische \\ Gesundheit, Medizinische Fakultät Mannheim, Universität Heidelberg, Mannheim, Deutschland \\ ${ }^{6}$ PONS Zentrum, Charité Mental Health, Klinik für Psychiatrie und Psychotherapie, Campus Charité Mitte, \\ Berlin, Deutschland \\ ${ }^{7}$ Centre for Population Neuroscience and Stratified Medicine (PONS), ISTBI, Fudan University, Shanghai, \\ China \\ ${ }^{8}$ Sozial- und Präventivmedizin, Department Sport- und Gesundheitswissenschaften, Strukturbereich \\ Kognitionswissenschaften, und Fakultät für Gesundheitswissenschaften Brandenburg, Profilbereich für \\ Versorgungsforschung mit Schwerpunkt eHealth, Universität Potsdam, Potsdam, Deutschland
}

\title{
Kohorten in der psychiatrischen Forschung
}

langen Zeitraum erfasst wird. Frühe Traumatisierungen haben schwerwiegende Auswirkungen auf die psychische Gesundheit im Jugend- und Erwachsenenalter. Daher sollte zumindest für derartige Fragestellungen die Untersuchung bereits bei der Geburt beginnen oder sogar intrauterine Belastungsfaktoren wie Rauchen oder Alkoholkonsum miterfassen [3].

\section{》) Neue Erkenntnisse liefern standardisierte Datenerhebungen in unterschiedlichen Kontinenten}

Im vorliegenden Schwerpunktheft von Der Nervenarzt werden unterschiedliche Kohorten und die hier erhobenen Erfahrungen und Herausforderungen diskutiert. Dies ist von großer aktueller Relevanz, da in diesen Tagen die Auswahl der Standorte für ein Deutsches Zentrum für Psychische Gesundheit (DZPG) erfolgt. Die Gründung des DZPG bietet die außerordentliche Gelegenheit, bestehende prospektive Kohorten zu koordinieren und fortzuführen und gleichzeitig durch die Bildung neuer Kohorten wichtige Bereiche der Gesundheitsvorsorge, die bis- her nicht hinreichend erforscht sind, zu stärken.

Adorjan et al. schildern in ihrem Beitrag „Neurogenetik der Schizophrenie: Erkenntnisse aus Studien basierend auf Datentausch und globalen Partnerschaften" entsprechend Untersuchungen bezüglich des Auftretens und der Ausprägung schizophrener Psychosen. Von besonderer Bedeutung hier ist die Erhebung molekulargenetischer und umweltbezogener Daten in unterschiedlichen Kontinenten, die es auch erlaubt, Umweltfaktoren zu identifizieren, die außerhalb der relativ gut beforschten europäischen und amerikanischen Lebenswelten zur Entstehung psychotischer Erfahrungen beitragen [4].

In der Arbeit mit dem Titel „Kohortenstudien in der Kinder- und Jungendpsychiatrie“ werden von Holz et al. Entwicklungsaspekte von Kinder und Jugendlichen diskutiert. Die Autoren verweisen hier insbesondere auf internationale longitudinale Studien, die sie in einer exemplarischen Beschreibung mit der der Mannheimer Risikokinderstudie vergleichen. Letztere untersucht ihre Probanden seit über 30 Jahren vom Zeitpunkt der Geburt an und hat wichtige Erkenntnisse zur Bedeutung der sozioökonomischen Ressourcen, des Stavoraussetzt, die über einen möglichst 
tus und geschlechtsspezifischer Effekte für das Auftreten psychischer Probleme in bestimmten Altersperioden geliefert. Dazu passend beschreiben Rapp et al. Potenziale und Grenzen von Alterskohortenstudien für die Gerontopsychiatrie. Auch hier geht es um die Erfassung spezifischer Mechanismen der Entstehung psychischer Erkrankungen in einem bestimmten Altersabschnitt, aus denen sich dann Präventionsansätze für psychische Erkrankungen ableiten lassen. Hier besteht allerdings ein relativer Mangel an klinischen Kohortenstudien, sodass hier der künftige Forschungsbedarf besonders deutlich wird.

\section{\) Über Satellitenortung lässt sich Verhalten im sozialen Kontext erfassen}

Internationale Aspekte der Forschung, die bereits in der Arbeit von Adorjan et $a l$. angeklungen sind, werden im Folgenden in den Arbeiten von Heinz et al. zur IMAGEN-Kohorte und von Schumann et al. zu populationsneurowissenschaftlichen Strategien zur Erhebung und Analyse klinischer und globaler Kohorten in den STRATIFY- und GIGA-Konsortien diskutiert. Die IMAGEN-Kohorte ist eine in Europa erhobene Gruppe von über 2000 jungen Menschen, die erstmals im Alter von 14 Jahren untersucht wurden und mittlerweile seit über 8 Jahren in weiteren Beobachtungszyklen nachuntersucht werden konnten. Am Beispiel der IMAGEN-Kohorte zeigen Heinz et al., dass sich sowohl nosologieübergreifende wie krankheitsspezifische Störungen im Bereich der Belohnungserwartung und der damit verbundenen neurobiologischen Systeme nachweisen lassen, die für die Entstehung von Suchterkrankungen, aber auch für affektive Störungen und Psychosen relevant sind. Schumann et al. erläutern, wie derzeit durch internationale Standardisierung und Angleichung der Erhebungsinstrumente Kernbefunde aus der IMAGENKohorte in unterschiedlichen Kontinenten überprüft, differenziert und erweitert werden können [5]. Von besonderer Relevanz sind hier neuartige Technologien, die wie beispielsweise die Satellitenortung das Verhalten der Probanden im jeweiligen sozialen Kontext direkt erfassen können. Hierbei ergeben sich allerdings komplexe datenschutzrechtliche Aspekte, die insbesondere dann von zusätzlicher Problematik sind, wenn die Untersuchungen in unterschiedlichen politischen Systemen stattfinden [6]. Auf diese ethisch wie datenschutzrechtlich relevanten Aspekte gehen die genannten Arbeiten vertieft ein.

Mit der internationalen Vernetzung von Kohortenstudien ergibt sich so erstmals die Möglichkeit, die Bedeutung sozialer Faktoren für die Entstehung und Aufrechterhaltung psychischer Erkrankungen in ihrem Wechselspiel mit genetischer Vulnerabilität und kulturellen Besonderheiten in verschiedenen Regionen der Welt zu untersuchen. Dadurch werden Erkenntnisse vertieft, wie auch auf Ebene sozialer Interventionen präventiv und therapieunterstützend gehandelt werden kann. Es bleibt zu hoffen, dass die genannten Themen eine zentrale Stellung im künftigen Deutschen Zentrum für Psychische Gesundheit einnehmen können.

Prof. Dr. Dr. Andreas Heinz

Dr. Kristina Adorjan

Prof. Dr. Dr. Tobias Banaschewski

Prof. Dr. Gunter Schumann

Prof. Dr. Dr. Michael Rapp

\section{Korrespondenzadresse}

Prof. Dr. med. Dr. phil. Andreas Heinz

Klinik für Psychiatrie und Psychotherapie, CCM, Charité - Universitätsmedizin Berlin

Charitéplatz 1, 10117 Berlin, Deutschland

andreas.heinz@charite.de

Interessenkonflikt. T. Banaschewski war innerhalb der letzten drei Jahre als Berater für die Firmen Lundbeck, Medice, Neurim Pharmaceuticals, Oberberg-GmbH, Takeda und Infectopharm tätig und hat Vortragshonorare von den Firmen Lilly, Medice und Takeda erhalten. Die vorliegende Publikation steht damit nicht im Zusammenhang. A. Heinz, K. Adorjan, G. Schumann und M. Rapp geben an, dass kein Interessenkonflikt besteht.

Open Access. Dieser Artikel wird unter der Creative Commons Namensnennung 4.0 International Lizenz veröffentlicht, welche die Nutzung, Vervielfältigung, Bearbeitung, Verbreitung und Wiedergabe in jeglichem Medium und Format erlaubt, sofern Sie den/die ursprünglichen Autor(en) und die Quelle ordnungsgemäßnennen, einen Link zur Creative Commons Lizenz beifügen und angeben, ob Änderungen vorgenommen wurden.

Die in diesem Artikel enthaltenen Bilder und sonstiges Drittmaterial unterliegen ebenfalls der genannten Creative Commons Lizenz, sofern sich aus der Abbildungslegende nichts anderes ergibt. Sofern das betreffende Material nicht unter der genannten Creative Commons Lizenz steht und die betreffende Handlung nicht nach gesetzlichen Vorschriften erlaubt ist, ist für die oben aufgeführten Weiterverwendungen des $\mathrm{Ma}$ terials die Einwilligung des jeweiligen Rechteinhabers einzuholen.

Weitere Details zur Lizenz entnehmen Sie bitte der Lizenzinformation aufhttp://creativecommons.org/ licenses/by/4.0/deed.de.

\section{Literatur}

1. Heinz A, Zhao X, Liu S (2020) Implications of the association of social exclusion with mental health. JAMA Psychiatry 77(2):113

2. Mascarell Maričić L, Walter H, Rosenthal A, Ripke $S$, Quinlan EB, Banaschewski T, Barker GJ, Bokde ALW, Bromberg U, BüchelC, Desrivières $S$, Flor H, Frouin V, Garavan H, Itterman B, Martinot JL, Martinot MP, Nees F, Orfanos DP, Paus T, Poustka L, Hohmann S, Smolka MN, Fröhner JH, Whelan R, Kaminski J, Schumann G, Heinz A, IMAGEN consortium (2020) The IMAGEN study: a decade of imaging genetics in adolescents. Mol Psychiatry 25(11):2648-2671

3. Heim C, Entringer S, Buss C (2019) Translating basic research knowledge on the biological embedding of early-life stress into novel approaches for the developmental programming of lifelong health. Psychoneuroendocrinology 105:123-137

4. Adorjan K, Odenwald M, Widmann M, Tesfaye M, Tessema F, Toennes S, Suleman S, Papiol S, Soboka M, Mekonnen Z, Rockstroh B, Rietschel M, Pogarell O, Susser E, Schulze TG (2017) Khat use and occurrence of psychotic symptoms in the general male population in Southwestern Ethiopia: evidence for sensitization by traumatic experiences. World Psychiatry 16(3):323. https:// doi.org/10.1002/wps.20470

5. Quinlan EB, Banaschewski T, Barker GJ, Bokde ALW, Bromberg U, BüchelC, Desrivières S, Flor H, Frouin V, Garavan H, Heinz A, Brühl R, Martinot JL, Paillère Martinot ML, Nees F, Orfanos DP, Paus T, Poustka L, Hohmann S, Smolka MN, Fröhner JH, Walter H, Whelan R, Schumann G, IMAGEN Consortium (2020) Identifying biological markers for improved precision medicine in psychiatry. Mol Psychiatry 25(2):243-253

6. Liu S, Müller S, Dolan RJ, Zhao X, Zheng JC, Heinz A (2020)Opportunities, risks and challengesinglobal mental health and population neuroscience: a case of Sino-German cooperation. Eur Arch Psychiatry Clin Neurosci. https://doi.org/10.1007/s00406020-01176-1 